\section{Research Square}

\title{
Association between physical activity pain and injuries in children
}

João Paulo de Aguiar Greca ( $\square$ joaogreca@hotmail.com )

Brunel University London https://orcid.org/0000-0001-9254-7975

Thomas Korff

Brunel University London

Jennifer Ryan

Royal College of Surgeons in Ireland

Research article

Keywords: paediatric obesity, pain, injury, school children, physical activity

Posted Date: November 4th, 2019

DOI: https://doi.org/10.21203/rs.2.16774/v1

License: (c) (i) This work is licensed under a Creative Commons Attribution 4.0 International License.

Read Full License 


\section{Abstract}

Background Children are advised to engage in physical activity to improve health. However, recent literature suggests an association between weight bearing activities, e.g. walking, and excessive joint loading in lower limbs of children. Excessive stress in joints of children may lead to pain or even cause injuries. The aim of this study was to investigate the relationship between pain, injury and physical activity in children.Methods This study had a cross-sectional design. 112 children aged 8-12 yr. (68 with normal weight, 38 with overweight or obesity) participated in the study. Moderate and vigorous physical activity were assessed using accelerometry over 7 days. Questionnaires were used to report pain (over 7 days) and injuries (over 12 months). Linear regression and negative binomial models were used to examine association between moderate activity, vigorous activity, and pain and injuries, respectively. Models were adjusted for potential confounders. Effect modification according to weight status was explored.Results There was no association between moderate physical activity and pain $(\beta=0.0004,95 \%$ $\mathrm{Cl}-0.007$ to $0.008 ; p=0.915)$ or between vigorous physical activity and pain ( $\beta=0.0069,95 \% \mathrm{Cl}-0.08$ to $0.22 ; p=0.354$ ). Similarly, no association was found between moderate physical activity and injuries (Rate Ratio $[R R]=1.00,95 \% \mathrm{Cl} 0.99$ to $1.01 ; \mathrm{p}=0.995)$ or vigorous physical activity and injuries $(\mathrm{RR}=1.00,95 \% \mathrm{Cl}$ 0.99 to $1.02 ; p=0.798$ ). There was no evidence that associations between physical activity and pain or physical activity and injuries, respectively, differed according to weight status.Conclusions Although paediatric obesity is linked to a higher incidence of pain and injuries in children, this study did not find that moderate and vigorous physical activity are associated with pain or injuries in children, regardless of their weight status.

\section{Background}

Children are advised to engage in 60 minutes of moderate-to-vigorous physical activity (MVPA) daily to improve health [1]. Physical activity (PA) is a broad concept that is defined as any bodily movement generated by skeletal muscle that results in energy expenditure [2]. Therefore, PA can be accumulated in short bouts performed as part of the child's daily routine. However, evidence shows that many children do not achieve PA recommendations [3]. Pain and injuries may act as a barrier to participation in PA for some children [4]. In particular, weight-bearing activities, such as walking and running that are habitually performed, may result in pain and injury because of increased joint loading [5]. However, few studies have examined if participation in PA is associated with pain and injuries in children.

Silva et al. found that self-reported time in moderate intensity PA (MPA) was associated with a higher probability of reporting pain on neck, shoulders, low back, wrists, hips, knees and ankles/feet. The authors also found that more time spent in vigorous intensity PA (VPA) was significantly associated with a higher probability of reporting pain on shoulders, mid back, knees and ankles/feet [6]. Conversely, Swain et al. found that girls who reported reduced participation in MVPA were more likely to have back pain, headache and stomach-ache [7]. Boys who reported reduced participation in MVPA were more likely 
to have stomach-ache or headache [7]. A third study found that frequent participation in MPA was associated with lower odds of injury among boys, while frequent participation in VPA was associated with higher odds of injury among girls [8].

While these studies suggest that PA may be positively or negatively associated with pain or injuries they used subjective measures of PA, which likely are inaccurate at measuring duration and intensity of PA [9]. Only one study has examined the association between objectively measured PA and pain in children [10]. The authors found no association between PA intensities or sedentary behaviour and back pain crosssectionally or longitudinally. However, this study focused only on neck and back pain and did not look at the association between MPA and pain, or VPA and pain separately. Finally, while one study found an association between objectively assessed MVPA and injuries [11], the study assessed upper extremity injuries only.

There are a lack of studies investigating if objectively MPA or VPA is associated with pain or injuries in children. Further, although the association between PA, pain and injuries may be stronger among children with overweight (OW) or obesity $(\mathrm{OB})$ compared to those with normal weight, no study has examined this. Thus, the present study aimed to investigate if MPA and VPA are associated with pain and injuries in children and if these associations differ between children with and without OW and OB.

\section{Methods}

\section{Study design}

The study had a cross-sectional design. Data collection took place in London, England and occurred from December of 2016 until March of 2017.

\section{Participants}

A convenience sample of children aged 8-12 years, was identified from three schools in the London Borough of Hillingdon, England, over 18 months. The three schools together had approximately 1,095 students enrolled aged 8 to 12 years. Head teachers sent participant information sheets and consent forms to parents of potentially eligible children. Children returned consent forms signed by their parents at the first assessment. In addition to parents, children also provided written assent. The present study received ethical approval from the Department of Life Sciences Research Ethics Committee at Brunel University London (reference number 2440-MHR-Mar/2016-2773-2). Children were excluded if they had a disability or medical condition that prevented them from engaging in daily physical activities as assessed using the Physical Activity Readiness Questionnaire (PAR-Q) [12]. 


\section{Procedure}

Data were collected by a single researcher at schools where participants were recruited from. Data collection occurred over two days: 1) on the first day, participants had the opportunity to ask questions about the study and decline to participate, before accelerometers, PA diaries and instruction sheets about accelerometer usage were distributed; 2 ) after seven days, the accelerometers and the PA diaries were collected, anthropometric measurements were assessed, and questionnaires regarding pain, injury and socioeconomic status were completed by the participant.

\section{Body composition}

Stature was measured to the nearest $0.1 \mathrm{~cm}$ using a calibrated stadiometer (Charder HM200P Portstad Stadiometer) and body weight was assessed to the nearest $0.1 \mathrm{~kg}$ using a calibrated electronic weight scale (Seca, Hamburg, Germany). BMI was calculated as weight (in $\mathrm{kg}$ ) divided by stature (in $\mathrm{m}$ ) squared. Children were categorised as normal weight or OW/OB using the extended international (International Obesity Task Force) BMI cut-offs for thinness, OW and OB [13]. Circumferences of waist and hip were collected using a Gulick anthropometric tape (Creative Health Products, Plymouth, USA). Measurements of waist and hip circumferences were recorded to the nearest $0.1 \mathrm{~cm}$. Waist circumference was measured horizontally at the midpoint between the inferior border of the bottom rib and the top end of the iliac crest. Hip circumference was measured around the broadest portion of the buttocks with the tape positioned parallel to the floor [14]. Body fat was assessed using skinfold measurement. Skinfold measurement of the triceps and medial calf sites were collected on the right side of the body using a Harpenden Skinfold Caliper (Country Technologies). Body fat of participants was estimated by the relative body fat for girls and boys using specific equations proposed by Slaughter [15].

\section{Socioeconomic status}

Socioeconomic status of participants was assessed using the Family Affluence Scale [16]. The Family Affluence Scale is a questionnaire developed specifically for young students and it aims to reflect money expenditure of a family [17]. The questionnaire was updated in 2008 and was found to be reliable and suitable for students [16]. Essentially, the Family Affluence Scale explores socioeconomic inequalities by classifying a set of items that reflects a family's assets and consumption. The questionnaire considers items such as the number of cars that a family possesses, whether a child owns their own bedroom, the number of times that their family went on holidays during the past 12 months and the number of computers their family has. This questionnaire has been widely used in children $[18,19]$ and also in research exploring the occurrence of injuries and PA [20,21]. After children responded to the questionnaire a factor score from zero to nine was attained, this score was then subsequently 
categorised into tertiles representing low, middle and high affluence groups: 0 to 3 low, 4 to 6 middle and 7 to 9 high [16].

\section{Assessment of injury}

The definition of an injury is widely accepted as an "event that requires medical attention" [20-22]. In the present study participants were asked to report injury events that required medical attention from a doctor or a nurse over the past 12 months. The following question was asked: "During the past 12 months, how many times were you injured and had to be treated by a doctor or nurse?" Injury in children has been widely investigated using this question [21-23].

\section{Assessment of pain}

Paediatric pain has been previously described as a subjective issue and is commonly reported by healthy children [24]. Participants were asked to self-report any pain or discomfort, of the whole body, that they experienced over the seven days of testing using a visual analog scale from the validated Pediatric Pain Questionnaire $[25,26]$. This method consists of participants marking a point on a $100 \mathrm{~mm}$ horizontal line with several faces representing "no pain" to "severe pain" [27]. Visual analog scales have been widely recommended as the most appropriate method for assessing pain in children $[28,29]$. It is the most extensively validated method used for assessing pain in children and adolescents [30].

\section{Accelerometry}

PA was assessed using a triaxial ActiGraph wGT3X-BT accelerometer (Pensacola, USA). The ActiGraph wGT3X-BT monitor is a small $(4.6 \mathrm{~cm} \times 3.3 \mathrm{~cm} \times 1.5 \mathrm{~cm})$ and light-weight device $(19 \mathrm{~g})$. The placement of the PA monitor plays an important role when assessing PA. Participants were requested to wear the accelerometer around their waist at the right hip[31] for all waking hours except when swimming, showering or during other water activities. Participants were asked to wear it for seven days. Participants completed PA diaries indicating periods of times when they did not wear the accelerometer.

A 5-second epoch and sampling rate of $30 \mathrm{~Hz}$ was used in the present study as children tend to engage in different intensities of PA in very short bursts, i.e. lasting less than 15 seconds [32]. The Actilife $6 \AA$ software was used to download and process all data recorded with the accelerometers. An upper limit of 20,000 counts per minute was established as a threshold to avoid spurious data or monitor failure [32, 33]. Non-wear time was defined as 60 minutes or more of consecutive zero counts. Minimum wear time 
was at least three days of 500 minutes [34]. Cut-points were 3581 to 6129 counts per minute for MPA [35] and $\geq 6130$ counts per minute for VPA [35].

\section{Statistical analysis}

The distribution of variables was assessed using Q-Q (quantile-quantile) plots and histograms. Variables that were normally distributed were described using means and standard deviations. Variables with skewed distributions were described using medians and interquartile ranges. Categorical variables were presented as frequencies and percentages. To examine whether there was any difference between the number of pain episodes reported by children with and without OW/OB, an independent two-sample $t$-test was performed. To examine whether there was any difference between injury incidence in children with and without $\mathrm{OW} / \mathrm{OB}$, the Mann-Whitney Utest was performed.

We examined associations between PA (MPA and VPA) and pain using linear regression models with pain as the dependent variable. We examined associations between PA (MPA and VPA) and injuries using negative binomial models. Negative binomial models were fitted instead of Poisson models as there was evidence of overdispersion [36]. We firstly fitted unadjusted models before adjusting for potential confounding variables. Confounding variables have been defined in the literature as variables that affect both the independent and dependent variable leading to false associations [37]. Potential confounding variables were identified based on the literature. These variables were age, socioeconomic status, sex, waist, BMI, hip, and body fat. Additionally, injury was considered as a potential confounder of the relationship between PA and pain.

After fitting unadjusted models, we included potential confounding variables one at a time to see how the coefficient changed with the inclusion of a potential confounder. We decided a priori that variables that changed the coefficient by more than $5 \%$ would be included in final models. From this exploratory analysis, the coefficient for PA or injuries, respectively, did not change by $>5 \%$ when any potential confounder was included. However, we included age, sex, socioeconomic status, injuries and OW/OB in the adjusted linear regression model and age, sex, socioeconomic status and OW/OB in the adjusted negative binomial model to demonstrate that adjusting for potential confounders had no impact on the effect estimate. We did not include waist circumference, hip circumference and body fat percentage in adjusted models as they were closely related to weight status and did not change the regression coefficients. We additionally included MPA-by-OW/OB and VPA-by-OW/OB interaction terms, respectively, in adjusted models to examine if the association between PA and pain and PA and injuries differed according to weight status. Residual plots were examined following linear regressions to identify if 
assumptions of homoscedasticity, normality and linearity were violated. Statistical analyses were performed using the statistical software STATA (StataCorp LLC, College Station, Texas, USA), version 13.

\section{Results}

Of the 1,095 students, aged 8 to 12 years, 114 children consented to participate. No child was excluded from the study based on their response to the PAR-Q. Eight students were not included in the final analyses, as they failed to record at least three days of PA. Therefore, 106 children were included in final analyses. The characteristics of participants are described in Table 1. Mean (SD) MPA was 100.4 (49.8) $\mathrm{min} /$ day. Mean (SD) VPA was 33.3 (24.7) min/day. Median (IQR) pain was $0 \mathrm{~mm}$ (3.0) (out of $100 \mathrm{~mm}$ ) and median (IQR) number of injuries was 0 (1.1).

Sixty-eight children (64.2\%) had normal weight and 38 children (35.8\%) had OW/OB. No difference in pain was found between children with normal weight and OW/OB (mean [SD] 1.41 [1.91] vs 1.18 [1.77], $\mathrm{p}=0.535)$ Similarly, no difference in number of injuries was found between children with normal weight and OW/OB (median [IQR] 0 [0-5] vs 0 [0-6], $p=0.504$ ). With regards to MPA, no difference was found between children with and without OW/OB $(p=0.959)$. Likewise, no difference was found in VPA between children with and without OW/OB $(p=0.947)$.

Insert table 1 here

\section{Association between PA and pain}

Table 2 presents results for associations between PA and pain. There was no evidence that MPA was associated with pain in children $(\beta=0.0004,95 \% \mathrm{Cl}-0.007$ to $0.008 ; p=0.915)$ even after adjusting the model for age, sex, socioeconomic status, OW/OB and injuries $(\beta=-0.0005,95 \% \mathrm{Cl}-0.008$ to 0.007 ; $\mathrm{p}=0.887)$. The association between MPA and pain did not differ according to weight status as evidenced by the $p$-value for the MPA-by-OW/OB interaction term $(p=0.909)$. There was no evidence that VPA was associated with pain in children from unadjusted or adjusted analyses (adjusted $\beta=0.0054,95 \% \mathrm{Cl}-0.009$ to $0.020 ; p=0.468$ ). There was also no evidence that the association between VPA and pain differed according to weight status $(\mathrm{p}=0.881)$.

Insert table 2 here 


\section{Association between PA and injuries}

Table 3 presents associations between MPA and VPA, respectively, and the incidence of injuries in children. There was no association between MPA and the incidence of injuries (Rate Ratio (RR) $=1.00,95 \%$ $\mathrm{Cl} 0.99$ to $1.01 ; p=0.995$ ), even after adjustment for age, sex, socioeconomic status and OW/OB (adjusted $\mathrm{RR}=1.00,95 \% \mathrm{Cl} 0.99$ to $1.01 ; \mathrm{p}=0.995)$. There was also no evidence that the association between MPA and injuries differed according to weight status $(\mathrm{p}=0.597)$. Finally, VPA was not associated with injuries in children ( $R R=1.00,95 \% \mathrm{Cl} 0.99$ to 1.02; $\mathrm{p}=0.798)$, even after adjusting for potential confounders $(R R=1.00$, $95 \% \mathrm{Cl} 0.99$ to $1.02 ; \mathrm{p}=0.868)$. There was also no evidence that weight status modified the association between VPA and injuries $(p=0.735)$.

Insert table 3 here

\section{Discussion}

The purpose of this study was to investigate the association between PA, pain and injuries in children. We found that MPA and VPA were not associated with pain or injuries in children. The association between PA and pain, and PA and injuries, respectively, also did not differ between children with and without OW/OB.

This is the first study investigating if objectively measured moderate and vigorous PA are associated with whole body pain and injuries. Two studies investigated the association between subjective PA intensity and pain in children. Silva et al. [6] reported that more time spent in MPA was significantly associated with a higher probability of reporting pain on neck, shoulders, low back, wrists, hips, knees and ankles/feet. While more time spent in VPA was significantly associated with a higher probability of reporting pain on shoulders, mid back, knees and ankles/feet. Swain et al. [7] found that reduced participation in MVPA was associated with presence of back pain, headache and stomach-ache in girls and also associated with combined headache and stomach-ache or headache in boys. Only one study has investigated the association between objectively measured PA intensity and pain, specifically back and neck pain, in children [10]. Similarly to findings of the present investigation, Aartun et al. [10] found no association between PA and spinal pain cross-sectionally nor longitudinally in a sample of 906 children aged 11-15 years.

Lowry et al. [8] found that high frequency in self-reported MPA was associated with decreased odds of injury among boys, and medium and high frequency in self-reported VPA were associated with injuries among girls. Similarly to findings of the present study, Nauta et al. [11] found that objectively measured 
MVPA was not a predictor of acute upper extremity injury risk. However, this study did not separate associations between MPA, VPA and injury.

No study has assessed if the association between PA and pain or the association between PA and injuries differs according to the weight status of children. The literature shows that $\mathrm{OW}$ and $\mathrm{OB}$ are related to musculoskeletal pain in children [38]. Although we hypothesised that children with OW/OB may be more likely to experience pain during PA as a result of increased joint loading [39], the results of the present study do not support this hypothesis. This is even after considering different intensities of PA. It is possible that type of PA is associated with pain and injuries in children rather than intensity of PA. It is also possible that an association between PA, pain and injuries exists among children with obesity rather than OW. Our sample included only 5 children with obesity and thus we analysed children with OW and $\mathrm{OB}$ as one group. Studies are needed to examine if PA type and intensity is associated with pain and injuries among children with obesity.

There are limitations to this study that should be considered. Given the cross-sectional design it is not possible to determine if current PA is associated with future pain or injuries. Also, pain was measured over seven days. Measuring pain during or immediately after PA would allow examination between PA and acute pain. Children were asked to recall injuries over the past 12 months, which may be difficult to recall and result in inaccurate estimates of the number of injuries experienced. A larger sample and recruitment from a wider geographical area would allow better representation of the sample.

\section{Conclusions}

Findings from this study indicate that MPA and VPA are not associated with pain or injuries in children. Our results also suggest that the association of PA with pain and injuries does not differ between children with or without OW/OB.

\section{Abbreviations}

PA: physical activity; MVPA: moderate-to-vigorous physical activity; OB: obesity; OW: overweight; PAR-Q: Physical Activity Readiness Questionnaire; SES: socioeconomic status; BMI: body mass index; MPA: moderate physical activity; VPA: vigorous physical activity.

\section{Declarations}

\section{Ethics approval and consent to participate}


The present study received ethical approval from the Department of Life Sciences Research Ethics Committee at xxx xxx xxx (reference number xxx). Written consent was obtained from parents in addition to written assent from children prior to their participation in the study.

\section{Consent for publication}

Not applicable.

\section{Availability of data and materials}

The datasets used and/or analysed during the current study are available from the corresponding author on reasonable request.

\section{Competing interests}

The authors declare that they have no competing interests.

\section{Funding}

This research was sponsored by the Capes Foundation within the Ministry of Education, Brazil (grant $\mathrm{n}$. $x x x x x x / x x-x)$.

\section{Authors' contributions}

JG was responsible for recruitment, data collection and writing the manuscript. JR and TK supervised this research study, contributed to the study design, supervised statistical analyses and editing the manuscript. All authors read and approved the final manuscript.

\section{Acknowledgements}

The authors would like to thank head teachers, teachers, parents and children for their contribution during the data collection. 
Author's information

Address for correspondence: Dr João Paulo de Aguiar Greca. College of Health and Life Sciences, Brunel University London. E-mail: joaogreca@hotmail.com

\section{References}

1. Department of Health Physical Activity Health Improvement and Protection. Start Active, Stay Active. 2011.

2. Caspersen CJ, Powell KE, Christenson GM. Physical activity, exercise, and physical fitness: definitions and distinctions for health-related research. Public Health Rep. 1985;100:126-31. doi:10.2307/20056429.

3. Hallal PC, Andersen LB, Bull FC, Guthold R, Haskell W, Ekelund U, et al. Global physical activity levels: Surveillance progress, pitfalls, and prospects. Lancet. 2012;380:247-57. doi:10.1016/S01406736(12)60646-1.

4. Smith SM, Sumar B, Dixon KA. Musculoskeletal pain in overweight and obese children. Int J Obes (Lond). 2014;38:11-5.

5. Greca JPA, Ryan J, Baltzopoulos V, Korff T. Biomechanical evaluation of walking and cycling in children. J Biomech. 2019;87:13-8. doi:10.1016/j.jbiomech.2019.01.051.

6. Silva AG, Sa-Couto P, Queiros A, Neto M, Rocha NP. Pain, pain intensity and pain disability in high school students are differently associated with physical activity, screening hours and sleep. BMC Musculoskelet Disord. 2017;18:194.

7. Swain MS, Henschke N, Kamper SJ, Gobina I, Ottová-Jordan V, Maher CG. Pain and moderate to vigorous physical activity in adolescence: An international population-based survey. Pain Med (United States). 2016;17:813-9. doi:10.1111/pme.12923.

8. Lowry R, Lee SM, Galuska DA, Fulton JE, Barrios LC, Kann L. Physical activity-related injury and body mass index among US high school students. J Phys Act Health. 2007;4:325-42.

9. Hidding LM, Chinapaw MJM, van Poppel MNM, Mokkink LB, Altenburg TM. An Updated Systematic Review of Childhood Physical Activity Questionnaires. Springer International Publishing; 2018. doi:10.1007/s40279-018-0987-0.

10. Aartun E, Hartvigsen J, Boyle E, Hestbaek L. No associations between objectively measured physical activity and spinal pain in 11-15-year-old Danes. Eur J Pain (United Kingdom). 2016;20:447-57.

11. Nauta J, Jespersen E, Verhagen E, van Mechelen W, Wedderkopp N. Upper extremity injuries in Danish children aged 6-12, mechanisms, and risk factors. Scand J Med Sci Sports. 2017;27:93-8.

12. Shephard R. PAR-Q, Canadian Home Fitness Test and Exercise Screening Alternatives. Sport Med. 1988;5:185-95.

13. Cole TJ, Lobstein T. Extended international (IOTF) body mass index cut-offs for thinness, overweight and obesity. Pediatr Obes. 2012;7:284-94. 
14. World Health Organization. Waist Circumference and Waist-Hip Ratio: Report of a WHO Expert Consultation. World Heal Organ. 2008; December:8-11.

15. Slaughter MH, Lohman TG, Boileau RA, Horswill CA, Stillman RJ, van Loan MD, et al. Skinfold Equations for Estimation of Body Fatness in Children and Youth. Hum Biol. 1988;60:709-23.

16. Currie $C$, Molcho M, Boyce W, Holstein B, Torsheim T, Richter M. Researching health inequalities in adolescents: The development of the Health Behaviour in School-Aged Children (HBSC) Family Affluence Scale. Soc Sci Med. 2008;66:1429-36.

17. Currie C, Elton R, Todd J, Platt S. Indicators of socioeconomic status for adolescents: the WHO Health Behaviour in School-aged Children Survey. Health Educ Res. 1997;12:385-97. https://oup.silverchaircdn.com/oup/backfile/Content_public/Journal/her/12/3/10.1093/her/12.3.385/2/12-3-385.pdf? Expires $=1488794418 \&$ Signature $=$ HLCfBjcv56661 x6ut07KNPI4TdWQt6n-PrZSNpkfnju9ZWRoTXg7nwPXy3z77nbx-87PUb8Q4beN4jcyd-gf32Pn02hsBWUa4iZM8KVFPgcCTwVr. Accessed 2 Mar 2017.

18. Voráčová J, Sigmund E, Sigmundová D, Kalman M. Family Affluence and the Eating Habits of 11-to 15-Year-Old Czech Adolescents: HBSC 2002 and 2014. Int J Environ Res Public Health. 2016;24. doi:10.3390/ijerph13101034.

19. Frasquilho D, de Matos MG, Marques A, Gaspar T, Caldas-De-Almeida JM. Factors affecting the wellbeing of adolescents living with unemployed parents in times of economic recession: findings from the Portuguese HBSC study. Public Health. 2017;143:17-24. doi:10.1016/j.puhe.2016.10.003.

20. Pickett W. Cross-national Study of Fighting and Weapon Carrying as Determinants of Adolescent Injury. Pediatrics. 2005;116:e855-63. doi:10.1542/peds.2005-0607.

21. Warsh J, Pickett W, Janssen I. Are overweight and obese youth at increased risk for physical activity injuries? Obes Facts. 2010;3:225-30.

22. Pickett W, Molcho M, Simpson K, Janssen I, Kuntsche E, Mazur J, et al. Cross national study of injury and social determinants in adolescents. Inj Prev. 2005;11:213-8.

23. Addor V, Santos-Eggimann B. Population-based incidence of injuries among preschoolers. Eur J Pediatr. 1996;155:130-5.

24. Anthony KK, Schanberg LE. Pain in children with arthritis: a review of the current literature. Arthritis Rheum. 2003;49:272-9. doi:10.1002/art.11010.

25. Gragg RA, Rapoff MA, Danovsky MB, Lindsley C, Varni JW, Waldron SA, et al. Assessing chronic musculoskeletal pain associated with rheumatic disease: Further validation of the Pediatric Pain Questionnaire. J Pediatr Psychol. 1996;21:237-50.

26. Varni JW, Thompson KL, Hanson V. The Varni/Thompson Pediatric Pain Questionnaire. I. Chronic musculoskeletal pain in juvenile rheumatoid arthritis. Pain. 1987;28:27-38.

27. Cohen LL, Lemanek K, Blount RL, Dahlquist LM, Lim CS, Palermo TM, et al. Evidence-based Assessment of Pediatric Pain. J Pediatr Psychol. 2008;33:956-7. doi:10.1093/jpepsy/jsm103. 
28. Huguet A, Stinson JN, McGrath PJ. Measurement of self-reported pain intensity in children and adolescents. J Psychosom Res. 2010;68:329-36. doi:10.1016/j.jpsychores.2009.06.003.

29. Stinson JN, Kavanagh T, Yamada J, Gill N, Stevens B. Systematic review of the psychometric properties, interpretability and feasibility of self-report pain intensity measures for use in clinical trials in children and adolescents. Pain. 2006;125:143-57. doi:10.1016/j.pain.2006.05.006.

30. Rapoff MA. Pediatric measures of pain: The Pain Behavior Observation Method, Pain Coping Questionnaire (PCQ), and Pediatric Pain Questionnaire (PPQ). Arthritis Rheum. 2003;49:S90-5. doi:10.1002/art.11396.

31. Trost SG, Mciver KL, Pate RR. Conducting accelerometer-based activity assessments in field-based research. Med Sci Sports Exerc. 2005;37 11 SUPPL.:531-43.

doi:10.1249/01.mss.0000185657.86065.98.

32. Heil DP, Brage S, Rothney MP. Modeling physical activity outcomes from wearable monitors. Med Sci Sports Exerc. 2012;44 SUPPL. 1:50-60.

33. Haapala HL, Hirvensalo MH, Kulmala J, Hakonen H, Kankaanpää A, Laine K, et al. Changes in physical activity and sedentary time in the Finnish Schools on the Move program: a quasiexperimental study. Scand J Med Sci Sports. 2016;[Epub ahea:1-12. doi:10.1111/sms.12790.

34. Trost SG, Pate RR, Freedson PS, Sallis JF, Taylor WC. Using objective physical activity measures with youth: How many days of monitoring are needed? Med Sci Sports. 2000;32:426-31.

35. Mattocks C, Leary S, Ness A, Deere K, Saunders J, Tilling K, et al. Calibration of an accelerometer during free-living activities in children. Int J Pediatr Obes. 2007;2:218-26.

doi:10.1080/17477160701408809.

36. Greene W. Functional forms for the negative binomial model for count data. Econ Lett. 2008;99:58590.

37. VanderWeele TJ, Shpitser I. On the definition of a confounder. Ann Stat. 2013;41:196-220.

38. Stovitz SD, Pardee PE, Vazquez G, Duval S, Schwimmer JB. Musculoskeletal pain in obese children and adolescents. Acta Paediatr. 2008;97:489-93.

39. Lerner ZF, Board WJ, Browning RC. Pediatric obesity and walking duration increase medial tibiofemoral compartment contact forces. J Orthop Res. 2016;34:97-105.

\section{Tables}

Table 1 Characteristics of participants. 


\begin{tabular}{|c|c|c|c|c|c|c|}
\hline & \multicolumn{2}{|c|}{ Normal weight $n=68$} & \multicolumn{2}{|c|}{ OW/OB $n=38$} & \multicolumn{2}{|c|}{ Total $\mathrm{n}=106$} \\
\hline & Mean & (SD) & Mean & (SD) & Mean & (SD) \\
\hline Age (years) & 10.4 & 1.3 & 10.4 & 1.1 & 10.4 & 1.2 \\
\hline Body mass (kg) & 34.5 & 7.8 & 49.3 & 10.3 & 39.5 & 11.4 \\
\hline Stature (cm) & 142.2 & 10.5 & 147.2 & 11.8 & 143.8 & 11.2 \\
\hline BMI $\left(\mathrm{kg} / \mathrm{m}^{2}\right)$ & 16.9 & 2.1 & 22.5 & 8.6 & 18.8 & 3.4 \\
\hline Waist circumference (cm) & 54.2 & 16.5 & 61.7 & 20.6 & 56.2 & 18.4 \\
\hline Hip circumference (cm) & 62.0 & 19.5 & 68.0 & 23.4 & 63.5 & 21.3 \\
\hline Pain (over the past seven days) ${ }^{a}$ & 0.0 & 3.0 & 0.0 & 3.0 & 0.0 & 3.0 \\
\hline Injury (over the past 12 months) ${ }^{\mathrm{a}}$ & 0.0 & 0.9 & 0.0 & 1.2 & 0.0 & 1.1 \\
\hline Body fat (\%) & 22.1 & 6.0 & 33.1 & 8.6 & 25.9 & 8.9 \\
\hline MPA (min/day) & 100.6 & 48.4 & 100.1 & 53.0 & 100.4 & 49.8 \\
\hline VPA (min/day) & 33.4 & 26.2 & 33.1 & 22.1 & 33.3 & 24.7 \\
\hline \multicolumn{7}{|l|}{ Socioeconomic status (\%) } \\
\hline Low & 13.9 & & 11.9 & & 13.2 & \\
\hline Middle & 54.2 & & 40.5 & & 49.1 & \\
\hline High & 31.9 & & 47.6 & & 37.7 & \\
\hline
\end{tabular}

${ }^{\mathrm{a}}=$ Median and interquartile range. SD: Standard deviation. Pain score 0 to 10. Injury = Number of injuries over the past 12 months.

OW/OB: Overweight/obesity.

Table 2 Unadjusted and adjusted associations between physical activity and pain in children.

\begin{tabular}{ccccccc} 
& Unadjusted $\boldsymbol{\beta}$ & $\mathbf{9 5 \%}$ CI & $\boldsymbol{p}$ & Adjusted $^{\mathrm{a}} \boldsymbol{\beta}$ & $\mathbf{9 5 \%} \mathrm{CI}$ & $\boldsymbol{p}$ \\
\hline MPA & 0.0004 & $(-.007$ to .008$)$ & 0.915 & -0.0005 & $(-.008$ to .007$)$ & 0.887 \\
VPA & 0.0069 & $(-.008$ to .022$)$ & 0.354 & 0.0054 & $(-.009$ to .020) & 0.468
\end{tabular}

adjusted for age, sex, socioeconomic status, injury and overweight/obesity.

MPA: moderate physical activity; VPA: vigorous physical activity

Table 3 Unadjusted and adjusted associations between physical activity and number of injuries in the past 12 months in children.

\begin{tabular}{lcccccc} 
& Unadjusted RR & $\mathbf{9 5 \%}$ CI & $\boldsymbol{p}$ & Adjusted $^{\mathrm{a}} \mathrm{RR}$ & $\mathbf{9 5 \%} \mathrm{CI}$ & $\boldsymbol{p}$ \\
\hline MPA & 1.00 & $(.99$ to 1.01$)$ & 0.995 & 1.00 & $(.99$ to 1.01$)$ & 0.995 \\
VPA & 1.00 & $(.99$ to 1.02$)$ & 0.798 & 1.00 & $(.99$ to 1.02$)$ & 0.868
\end{tabular}

aAdjusted for age, sex, socioeconomic status, and overweight/obesity.

MPA: moderate physical activity; VPA: vigorous physical activity 